\title{
A review of Anti-bacterial activity of Nigella sativa in gut of broiler chicks
}

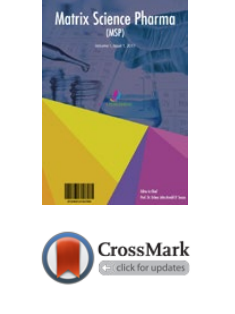

Institute of Pharmacy, Physiology \& Pharmacology,University of Agriculture, 38040 Faisalabad, Pakistan

This is an open access article distributed under the Creative Commons Attribution License, which permits unrestricted use, distribution, and reproduction in any medium, provided the original work is properly cited.

\section{ARTICLE DETAILS}

Article history:

Received 22 January 2017

Accepted 03 February 2017

Available online 05 February 2017

Keywords:

Anti-bacterial, Thymoquinone, Nigella sativa, Feed Conversion Ratio, Immune Titer.

\section{ABSTRACT}

Nigella sativa (N. sativa) is an important herb with multiple pharmacological activities. Therapeutic properties of N. sativa based on the availability of thymoquinone (TQ) which is an essential oil and an active chemical component. This review concerns about the anti-bacterial activity of N. sativa in the gut of broiler chicks. The addition of $\mathrm{N}$. sativa powder appeared to have a positive impact on growth performance of broiler chicks as it improved body weight gain and feed conversion ratio (FCR) of broilers at finisher phase and it could be considered as a substitute of antibiotic growth promoter (lincomycin) for broiler chicks. It also affect the immune titer of Infectious Bursal Disease and New Castle Disease in the broiler chicks.

\section{Introduction}

For many years, antibacterial feed additives are widely used, to improve the production in terms of feed conversion ratio and weight gain. The growth performance of broiler chicks can be improved by controlling the infections in the gut mucosa. As resistance to these antibacterial substances is high, therefore the use of these feed additives in animals and poultry feed was banned throughout the world in 2006.Therefore, there is need to use some alternatives such as, from plant origin, for example plant extracts, essential oils and whole plant as antibacterial feed additives (Mufarrej, 2014).

Increasing cost of meat is most declining factor in the poultry industry. Foodstuff cost constituted the $80 \%$ of total production cost in the poultry industry. Therefore, high cost of foodstuff is the main factor in increasing the cost of broiler meat. Because of antibiotic resistance and their residues, poultry industry is looking for natural alternatives. To increase the growth rate and feed efficiency of broiler, research is being conducted on natural feed additives like Nigella sativa (N. sativa) (Dieyeh and Mohammad 2008). Now a day, growing public health problem is the development of bacterial resistance due to excessive use of antibiotics. Excessive exposure to antibiotics is the main reason for development of antibiotic resistance. In both animals and humans, it is same. Source of antibiotic exposure in human is animal husbandry and food additives. This results in the development of resistant bacterial strains. In fact, there are two types of antibiotic resistance, in one type, resistance develops when bacteria resist to antibiotics due to their natural ability of resistance to a certain type of antibiotics (Alcaine et al., 2015). The mechanism behind this resistance is inactivation of enzymes. This resistance type develops in the enzyme's presence. For example, Staphylococcus that produces an enzyme penicillinase, this enzyme is capable of breaking the penicillin's molecular structure and results in resistance (Apata, 2009).

However, for promoting growth in farm animals, these feed antibiotics have shown negative results on animal's profitability. Research being conducted on various active substitutes for antibiotics for example black cumin. Black cumin is also known as black seeds or N. sativa. Black seeds are mostly grown in Mediterranean and Asian countries (Mufarrej, 2014). Nigella sativa (N. sativa) is a medicinal plant, belongs to Family Ranunculacea, is an emerging miracle herb having a rich historical background. N. sativa is a flowering plant that grows annually up to $20-90 \mathrm{~cm}$ tall. Leaves of $\mathrm{N}$. sativa segmented, thread like to narrowly linear. Endosperm of N. sativa is composed of thin walled cells that are rectangular and polygonal; these cells are filled with oil globules (Salam, 2012). Microscopic view of N. sativa seedpowder shows that these are parencymatous cells with oil globules in it (Safhia et al., 2014). N. sativa is much richer in active compounds (Bourgou et al., 2008).

\section{REVIEW OF LITERATURE}

The fastest growing source of meat and eggs in the world is poultry. Through this poultry source about one fourth of all meat produced during the year
2000. The market ready broiler can produce in less than six weeks through the modern production unit. This was possible by the genetic selection that results in improved health and feeding management. In intensive farming systems, therapeutic agents and antibiotics treat bacterial diseases. These therapeutic agents used as growth promoters as well as prophylactic agents in the feed and water of birds for example bacitracin, neomycin, oxytetracycline, avoparcin and lincomycin (Apata, 2009).

These growth promoters and antibiotics used at sub-therapeutic dose to improve the productivity in terms of converting feed to meat and rate of weight gain. In 1950's, the suggested dose of therapeutic agents in the feed was $5-10 \mathrm{~g}$ per kilogram and since then it has increased by 10-20 folds. Antibiotics mostly used in many developing countries to treat the infections and to lessen the adverse effects of stress responses in poultry. Antibiotic usage hasmodernized livestock and poultry production resulted in advantages of health and economic (Apata, 2009).

Foodstuff is a part of high financial significance in the raising of poultry's business not only because of it is responsible for the essential growth of birds, but most importantly, it shows the largest expense in the development cycle. For example, the energy requirement for broiler is responsible for $70 \%$ of the cost. In addition, grains type and processing method interfere in a different way on animal performance and viability. The benefits of utilizing the processed foodstuff have well reported despite of the fact that they have high manufacturing cost. Under normal conditions, winged creatures need to manage diverse sorts of food, which have diverse protein and energy levels (Neves et al., 2014).

Researchers have linked the behavior and performance of broiler chicken to physiological responses and productivity. However, there was a deficiency of controlled information of the biomechanical structures and features of the feeding process of birds (Neves et al., 2014).

Generally, sensitive bacterial strains were eliminated by antibiotics upon application in poultry farming, but some variants were left behind with unusual traits resulted in resistance. These resistant strains were multiplied and increased to million fold, became predominant microorganism's population (Gould, 2008).

The major public health problems has been appeared from pathogenic bacteria that developed the antibiotic resistance, that has led to increase discussion related to careful use of antimicrobial substances particularly medicine used in veterinary, agriculture and nutrition. Since antimicrobial agents used in treatment, prophylaxis and for growth promotion, their usage had played an important role in animal husbandry (Bogaard et al., 2002).

Generally, in poultry chicks and animal husbandry, large numbers of antimicrobial substances have been using as prophylaxis and in the treatment of several diseases as well. Regardless of the health status of animals, there is an excessive exposure of animals to a sub-therapeutic dose of 
antimicrobial agents. Such exposure has resulted in the development of resistant bacterial strains. For decade, a selective pressure has exerted to target bacteria by antibiotics used in animals that closely related to human treatment (Castanon, 2007).

If antimicrobial resistant bacteria in food animals became the part of the human bacterial population, they will threaten the drug's efficacy for human use. For example, Salmonella resistance developed due to highfrequency use of antibiotic in agriculture. Generally, resistance to infection of Salmonella developed due to eating polluted food from animal's origin (Oliveiraa et al., 2005).

\subsection{Micro biota of gut, defense system and bird's performance}

It was reported that, the composition of bird's immune system consist of several cells and soluble factors that makes it very complex system like mammal's immune system. In order to produce the protective immune response, soluble factors and several cells worked together. It has found that for the development and maturation of immune responses (innate and adaptive immune responses) of chicken commensally gut micro biota is an important inducer. It has suggested that based on studies in mammals, certain immune cell's population have accumulated in poultry intestine through specific species of bacteria. Bacteroides fragilis (belonging to the phylum Bacteroidetes) reported as an example. Interleukin-17 has shown to produce by bacteria Bacteroidesfragilis. Another example of bacteria i.e. Lactobacillithat long been known for their capability for activation of immune system of intestine that resulting in increased resistance to various diseases, because it induces a peptide of low molecular weight that activates the immunity. It has reported that these bacteria have capability to produce the short chain fatty acids (SCFAs) (Sugiharto, 2014).

These fatty acids were bacteriostatic for certain types of bacterial species; they inhibit the growth of bacteria either directly or through lessening the $\mathrm{pH}$ of the intestinal environment. Researchers have found that colonization resistance against pathogenic bacteria produced through bacteriocins by modifying the receptors utilized by these pathogenic bacteria. Renewal and barrier function of gastrointestinal epithelium favored by short chain fatty acids produced by lactic acid bacteria. Different studies have suggested that intestine contain both beneficial for health and pathogenic bacteria, for example, lactobacilli and bifidobacteria (gram-positive) and clostridium species, Salmonella, and Escherichia coli (Sugiharto, 2014).

Poultry was a major reservoir of antibiotic resistant bacteria because of the extensive use of antibiotics in poultry industry. Therefore, antibiotic resistance has limited the use of available options of therapies in specific cases of infections caused by Salmonella. It also reported by the World Health Organization (WHO), that in case of infection in combination with Human Immunodeficiency Virus (HIV), it resulted in the rapid propagation of the disease. The Salmonella species have beensegregate in Brazil. However, world's second manufacturer of poultry meat is Brazil (Oliveiraa et al., 2005).

It was reported that the main risk factor for antibiotic resistance is their widespread use that resulted in increased number of resistant bacterial strains. Levels of resistance to antibiotics in bacteria were varying from bacterium to bacterium. For example, strains of Escherichia coli (E.coli), Enterococcus and Staphylococcus species were different in poultry flocks. In E.coli $97 \%$ strains have shown resistance to tetracycline, $31 \%$ to piperacillin and $51 \%$ to ampicillin (Alcaine et al., 2015).

It resulted in a huge number of bacteria resistant to antimicrobials. If antimicrobial resistant bacteria in food animals became the part of the bacterial population of human, they threaten the drug's efficacy of human use. For example, Salmonella resistance developed due to highfrequency use of antibiotic in agriculture. Generally, resistance to infection of Salmonella developed due to eating polluted food from animal's origin (Oliveiraa et al., 2005).

In the food animal production, increase the concentration of antibiotics used to treat and control of microbial diseases and increase growth rate. The development of resistant genes and resistant microbes attributed to high frequency use of antibiotics. It is possible in Denmark to decrease the use of antibiotics in animals, which used for food purposes that significantly causes the low resistance. Use prudent antibiotics can help to decrease the resistance and based on the knowledge and the susceptibility of the microbes and human problems. Following parameters may decrease the development of resistance like; knowledge about the development of antibiotic resistance in livestock used for food production, the impact of usage of many antibiotics on resistance and the suitable treatment to control resistance development (Apata, 2009).
An antibiotic agent is mostly used in livestock production and in human medicine for greater than 60 years improving animal and human health, but can promote the spread and development of antibiotic resistant pathogens all over the world. These reports are emphasis on the commercial value of the antibiotic growth promoters (AGPs) to both consumers and producers. This report explores the potential adverse effects of limiting the antibiotic, after calculating the magnitude of modern antibiotic consumption in animals worldwide, which can increase the growth rate of animals. The antimicrobial growth promoters could be restricted in well-developed industrialized nations, but is potentially greater in underdeveloped countries with less developed production practices and hygiene. With no main changes in policy, worldwide consumption of antibiotic in animals that used for human consumption is likely to rise by two-thirds by 2030, with the common of that increase occurring in demand for animal products, particularly in poultry (Proudfoot et al., 2007).

Ofloxacin and ciprofloxacin have reported with 10 percent of resistant strains. Erythromycin has shown $39 \%$ resistant strains, 19\% clindamycin, $13 \%$ ofloxacin and $14 \%$ tetracycline in staphylococcus. It has observed that tetracycline, nitrofurantoin and erythromycin have $80 \%, 34 \%$ and $59 \%$ resistance in enterococci, respectively. It was found that the high incidence of resistant strains in poultry puts the public health at risk (Apata, 2009).

\subsection{Lincomycin as a growth promoter}

Lincomycin belongs to an antibiotic class. Itis produce by Streptomyces lincolnensis var. lincolnesis. There are many uses of lincomycin, for example, it used in the treatment of staphylococcal, streptococcal, and Bacteroidesfragilis infections. It has shown the activity against gram positive, gram negative and anaerobic bacteria. It also showed the activity against these organisms; Aerobic gram-positive cocci: Streptococcus pyogenes and Viridans group streptococci; Aerobic gram-positive bacilli: Corynebacterium diphtheriae; Anaerobic gram-positive non-sporeforming bacilli: Propionibacterium acnes; Anaerobic gram-positive sporeforming bacilli: Clostridium tetani and Clostridium perfringens (Proudfoot et al., 2007).

Table 2.1: Chemical properties of lincomycin

Chemical name Lincomycin: Lincomicina: Lincomycinum

Molecular formula $\quad$ C18H34N206S

Molecular weight $\quad 406.53736 \mathrm{~g} / \mathrm{mol}$

\section{(Morar et al, 2009).}

\subsection{Structure of lincomycin}

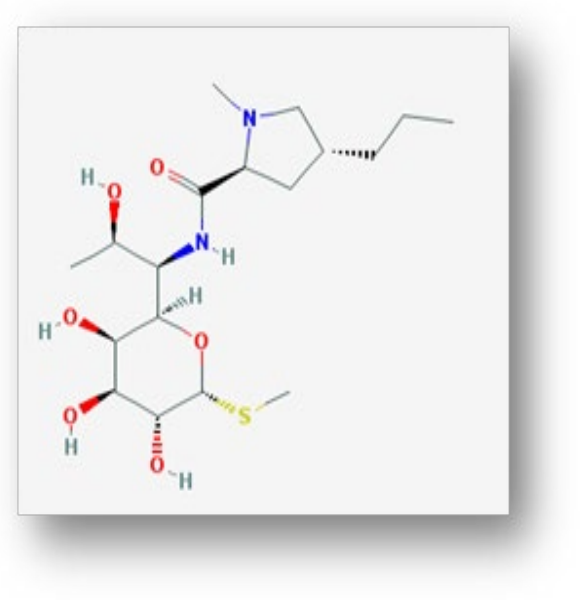


Fig 2.1: Structure of Lincomycin (Proudfoot et al., 2007)

Structure was prepared by using Chem Draw ultra

Effects of lincomycin in male broiler chicks were determined through an experiment in which lincomycin at the dose of $2.2 \mathrm{mg} / \mathrm{kggave}$ either in food or in drinking water (almost $0.5 \mathrm{ml}$ or equivalent amount). The experiment was comprised of total four treatments. One was the control group without lincomycin;the second group was containing $2.2 \mathrm{mg}$ of lincomycin per kilogram, control diet plus drinking water containing lincomycin at different concentrations. Such concentrations have intended to provide an intake that is equivalent to treatment 2 , and treatment 14 with lincomycin concentration reduced by half.

There was no considerable effect observed in any treatment on mortality, food utilization efficiency and final body weight of broiler chicks. It concluded that the dose of lincomycin $2.2 \mathrm{mg} / \mathrm{kg}$ did not be more effective in improving the performance of broiler chicks both economically and biologically (Proudfoot et al., 2007).

\subsection{Mechanism of Action of Lincomycin}

\subsubsection{Protein Synthesis Inhibitor}

Lincomycin binds to the $50 \mathrm{~S}$ ribosomal subunit. It results in inhibition of protein synthesis. By this way, it produces the bactericidal effects in susceptible organisms. Antibacterial mechanism includes disruption of peptide chain elongation, genetic code misreading, blockage of A-site of ribosomes and oligosaccharides side chain's attachment to glycoprotein (Morar et al., 2009).

\subsubsection{Absorption, Distribution and Excretion}

Lincomycin absorption occurs in the gastrointestinal tract (GIT). Its absorption occurs rapidly but partially up to $20-30 \%$. After the oral administration of $500 \mathrm{mg}$ of lincomycin, the peak plasma concentration of lincomycin was $2-5 \mu \mathrm{g} / \mathrm{ml}$. It reported that antibiotic activity persists for about 12 hours for most of the gram-positive microorganism. The important route of excretion is bile and urine. The active form of the drug found to appear in feces after oral and parenteral administration. It was suggested its excretion through both intestinal wall and bile (Morar et al., 2009).

\subsubsection{Biological Half-Life}

The plasma half-life of lincomycin is 4-6.4 hours in patients with normal renal function. The plasma half-life is increase in proportion to the degree of impairment in patients with reduced renal or hepatic function. Total 100 Plasma half-lives as high as 3 times normal have been reported in patients with severe renal impairment. The half-life may be two times normal in patients with hepatic impairment (Morar et al., 2009).

\subsubsection{Metabolism/Metabolites}

Lincomycin partially metabolized in the liver, both drug, and metabolites excreted in urine, bile and feces (Morar et al., 2009).

\subsection{Pharmacology of Lincomycin in Animals}

Effectiveness of lincomycin (LINCOCIN) studied in experimental animals in vivo. Those animals were infected with Streptococcus viridans, $\beta$-hemolytic Streptococcus, Staphylococcus aureus, Diplococcus pneumoniae and Leptospirapomona. Infectiveness found against Klebsiella, Pasteurella, Pseudomonas, Salmonella and Shigella infections (Morar et al., 2009).

\subsection{Nigella sativa and its application in poultry}

From botanical family Ranunucleae black seed called Nigella sativa. Chemical composition of N. sativa is in the following table:

Table 2.2: Chemical composition of $N$. sativa and their amount present in black seed

\begin{tabular}{|c|c|}
\hline Constituent & Percentage amount \\
\hline Thymoquinone & $30 \%-48 \%$ \\
\hline p-cymen & $7 \%-15 \%$ \\
\hline 4 -terpinol & $2 \%-7 \%$ \\
\hline t-anethol & $1 \%-4 \%$ \\
\hline Linoleic acid & $50 \%-60 \%$ \\
\hline c-Sitosteral & $44 \%$ \\
\hline Fat & $28.5 \%$ \\
\hline Carvacol & $6 \%-12 \%$ \\
\hline Longifolene & $1 \%-8 \%$ \\
\hline Carbohydrates & $33.9 \%$ \\
\hline Proteins & $16-19.9 \%$ \\
\hline
\end{tabular}

(Bourgou et al., 2008; Ahmad et al., 2013).

The chemical composition of $\mathrm{N}$. sativa has shown that it is composed of fixed oils (36\%-38\%), essential oils (0.4\%-2.5\%) and saponins, proteins and alkaloids. Fixed oil mainly composed of unsaturated fatty acids, for example, arachidic and eicosadienoic acids. Burits and Bucar (2000) were two scientists who had analyzed the essential oils of N. sativa by using Gas Chromatography-Mass Spectrometry (GC-MS) (Ali and Gerald, 2003).

Volatile oil and fixed oil are two types of oils that also found in extracts of N. sativa. These essential oils contained thymoquinone (TQ), thymohydroquinone (THQ) and dithymoquinone (DTQ). Crystalline compound found to contain in seeds of N. sativa is known as thymoquinone/ nigellone. The protective effect of $\mathrm{N}$. sativa against diseases found due to thymoquinone or nigellone. The first identified active constituent was nigellone, from which thymoquinone was isolated. The process of dimerization occurred when thymoquinone exposed to air, as a result dithymoquinone compound was formed (Mady et al., 2013).

Among a long list of active constituents of N. sativa, thymoquinone was the major active component (27.8\%-57.0\%). Dithymoquinone rapidly dimerized by exposure of thymoquinone to air. It has reported that seed of N. sativa contains four alkaloids as constituents such as nigellicine, nigellidine having an indazole nucleus while nigellimine and its $\mathrm{N}$-oxide belongs to isoquinolines. Currently, a new compound was isolated from seeds of N. sativa is known as triterpene saponin alpha hederin. Previously this compound had reported in leaves of Hedera helix. It found that oil contains different types of compounds that act in synergistic manner. In pharmacological studies, this fact suppressed the importance of utilization of the whole seed's oil (Morar et al., 2009).

This property has observed before in various spices. However, the antioxidant activity of $\mathrm{N}$. sativa has not been involving the iron-complexing activity. Various studies have revealed that the basis of many human diseases was activity of free radicals, partially or completely, therefore, in folk medicine, the anti-oxidant activity of N. sativa may explain its efficacy (Burits and Bucar, 2000).

Anti-oxidant property of N. sativa has shown in hepatoxicity caused by Carbon Tetrachloride ( $\mathrm{CCl} 4)$, cirrhosis, liver damage due to Schistosoma mansoni infection and liver fibrosis. Researchers have proved that after performing three types of tests on mice and rats, they concluded that fixed oil of N. sativa has potential anti-nociceptive activity and this activity is due to opioid active constituent found in oil. It antagonized by naloxone (Burits and Bucar, 2000).

In addition to these uses, N. sativa has been used as a CNS depressant. Researchers have also reported the analgesic activity of N. sativa, because it contains thymoquinone, the major active principle. By using different types of antagonists of opioid like naloxone, and kappa, mu, delta receptor antagonists, the mechanism of analgesia reported. Through indirect activation of mu and kappa receptors of super spinal, N. sativa has found to produce anti-nociceptive and analgesic effects (Islam et al., 2015).

Experiments on mice conducted to prove the anti-inflammatory, analgesic and antipyretic action of N. sativa in humans. The mechanism through which N. sativa have shown its anti-inflammatory actions have studied. A potent inhibitor of eicosanoid family including thromboxane B2 and leukotrienes B4 was thymoquinone. Thymoquinone has shown to inhibit the cyclooxygenase and lipoxygenase in order to inhibit the thromboxane B2 and leukotrienes B4 respectively. However, it has shown that N. sativa oil has greater potential of anti-oxidant and anti-nociceptive activity as compared to thymoquinone (Bashir et al., 2015).

Some workers have investigated possible antitumor activities of crude and purified components of N. sativa. Such as, in the case of Erlich ascites carcinoma, Dalton's ascites lymphoma and sarcoma, while its crude methanol extract have shown a potential cytotoxicity activity. The same results have observed in mice suffered from Erlich ascites carcinoma that had to receive $2 \mathrm{mg}$ of the $\mathrm{N}$. sativa extract per mouse per day for 10 days. It also has observed that in Jukart lymphoma cells the cellular expression of specific polypeptides altered by exposure of $\mathrm{N}$. sativa to the volatile oil. 
compounds isolated from N. sativa. For example, two stages of skin cancer, initiation and promotion inhibited in mice by topical application of the extract of N. sativa. Experiments were conducted to induce soft tissue sarcomas by subcutaneous injections of 20-Methylcholantherene (MCA) in mice after that the researches have noted that the incidence of this sarcoma was lessened by intraperitoneal injection of N. sativa. Studies have revealed that therapy with crude ethanol extract considerably reduced the tumor induced by cisplatin. The mouse was treated with extract of N. sativa 30 minutes before cisplatin; protection against nephrotoxicity induced by cisplatin was observed (Majdalawieh et al., 2010).

It was uncertain, that protective mechanism is responsible for this action but it has proposed that either increase elimination of cisplatin or decrease in nephrotoxicity development. Thymoquinone in combination with ifosfamide (IFO) has given in drinking water at the rate of $5 \mathrm{mg}$ per kilogram per day for 5 days; it has resulted in attenuation of renal toxicity caused by ifosfamide (IFO). This was possible due to anti-oxidant activity of thymoquinone (Burits and Bucar, 2000).

Thymoquinone enhanced the anti-tumor activity of cisplatin. Cytotoxicity to various parental and multi-drug resistant human tumor cell line of crude gum, fixed oil and two components that were thymoquinone and dithymoquinone has tested. Gum and oil have not been associated with cytotoxicity while thymoquinone and dithymoquinone were cytotoxic to Multi-Drug Resistant (MDR) human cell lines. The N. sativa fraction known as ethyl acetate has a strong cytotoxic action against different cell lines in-vitro. Inhibitory affects of thymoquinone both in-vivo and in-vitro against 20- methylcholanthrene induced fibro sarcoma and pyrene induced for stomach cancer production were observed in mice. Thymoquinone administration has lessened the initiation of fibro sarcoma and rate of mortality in mice.

In vitro, it has observed that survival of fibro sarcoma was effective through thymoquinone. The mechanism behind it was uncertain, but it has shown that there was interference with synthesis of dioxyriboneuclic acid (DNA) associated with an increase in the process of detoxification. Most beneficial effects were shown on oxidative status and it was accomplished by production of the reduced form of glutathione, the activities of some enzymes (Jaswal et al., 2016).

\subsection{Hepatoprotective and Nephroprotective action}

It has shown that N. sativa sold to treat the liver conditions in some countries. An experiment conducted on isolated rat's hepatocyte. The protective effect of thymoquinone was observedagainst hepatic problem. This effect caused by tert-butyl hydroperoxide (TBHP). This hepatoprotective action of thymoquinone compared to a hepatoprotective agent known as silybin. Thymoquinone found to be more protective than silybin. The basis of hepatoprotective action was related to conservation of glutathione intracellularly, in the case of glutathione depletion oxidative stress have shown to increase and cells became more susceptible to irreversible injury. Inhibitory action of thymoquinone for the production of thromboxane B2 was also suggested the mechanism of hepatic protection (Jaswal et al., 2016).

Protection against D-galactosamine and carbon tetrachloride induced liver damage in rats observed that were pretreatment with N. sativa for 4 weeks. After oral administration of thymoquinone oil at a dose of $800 \mathrm{mg} /$ $\mathrm{kg} /$ day for 4 weeks, no hazardous effects seen on liver functions. Serum total cholesterol, low-density lipoproteins and triglycerides levels found to reduce by N. sativa oil. The literature has shown that pretreated rabbits with N. sativa prevented from liver cirrhosis and fibrosis. Histological pictures and oxidative status indices were improved by extract of N. sativa (Suddek, 2014).

The researchers have found that if thymoquinone was removed from N. sativa, it used as, potential respiratory stimulant. According to an experiment, performed on guinea pigs have proved the beneficial effects of N. sativa in asthmatic patients. An experiment in Pakistan have shown that the dose-dependent relaxation of spontaneous contraction of the jejunum of rabbits. Extract of N. sativa produces restriction of potassium chloride $(\mathrm{KCl})$ induced contraction of the jejunum of the rabbits. This effect was similar to that produced by verapamil (calcium channel antagonist). The basis of this mechanism of contraction was antagonism of contraction induced by $\mathrm{KCl}$, carbachol and histamine. Calcium channel found to be responsible for bronchodilator and spasmolytic action. Studies have revealed that 10 times higher pharmacological activity found in ether petroleum extract of black seeds as compared to those crude extract (Kaleem et al., 2006).

Thymoquinone and dithymoquinone found to have strong anti-histaminic activity. For confirmation of anti-histaminic effect, an experiment performed on rat peritoneal mast cells in vitro, using nigellone (carbonyl polymer of thymoquinone) purified from N. sativa. Nigellone was effective in inhibiting the histamine release (Shakeri et al., 2016).

\subsection{Nigella sativa and its Anti-diabetic action}

In an experimental rabbit, the effect of $\mathrm{N}$. sativa on alloxan-induced diabetes mellitus has reported. It has investigated that administration of N. sativa volatile oil intraperitoneally considerably reduced the fasting blood glucose level in both normal and hyperglycemic rabbits after 4-6 hours of administration. It has shown that there was no effect on insulin concentration with this treatment, so mechanism behind hypoglycemic activity does not seem to involve insulin. However, it has suggested that structural integrity of pancreatic cells in diabetic rats restored by $\mathrm{N}$. sativa. Histology of liver and pancreas and effects of plant extract on lipid peroxides, glucose and glutathione have investigated. Because of treatment for 2 months with this extract considerably lessened the high concentration of lipid peroxide and glucose. Levels of glutathione and ceruloplasmin have significantly reduced by N. sativa extract. Anti-oxidant property of black seeds was the basis of anti-diabetic effect (Kaleem et al., 2006).

\subsection{Effect on cardiovascular system and blood}

Effects of $\mathrm{N}$. sativa and its active constituent (thymoquinone) was investigated arterial blood pressure in anesthetized rats and on their heart. After intravenous injection of both these agents, there was a dose-dependent decrease in heart rate and arterial blood pressure. Their action antagonized by atropine, histamine receptor antagonist like cyproheptadine, adrenergic depleting agents (reserpine) and by spinal pithing. These agents' action found to be multifactorial and centrally mediated through an indirect and direct mechanism, for example, cholinergic and tryptaminergic mechanism. Oil could be use as centrally acting antihypertensive (Abbasnezhad et al., 2016).

It suggest that the $\mathrm{N}$. sativa oil has a methanol soluble portion that showed the inhibitory effects on arachidonic acid (AA) mediating aggregation of platelets and coagulation of blood. Several compounds were isolated from the oil having anticoagulant activity, more than that therapeutic agents used in thrombosis like aspirin. Worker have found that the extract of N. sativa with dichloromethane have potent diuretic and antihypertensive action. Its diuretic action compared with frusemide, and 16\% diuretic action found with extract of $\mathrm{N}$. sativa. Diuretic action related to increase elimination of sodium, chloride, potassium and urea. Decrease in arterial blood pressure through N. sativa found $22 \%$ as compared to calcium channel blockers with $18 \%$ decrease in arterial blood pressure (Kaleem et al., 2006).

\subsection{Antiulcer action}

Ulcer index in rats found to reduce by almost $36 \%$ through aqueous extract of N. sativa seeds. This extract decreased the peptic activity and production of acid, however did not alter the activity of mucin. More recently, opposite results observed that $\mathrm{N}$. sativa oil administration for 2 weeks enhanced mucin and glutathione contents in gastric and decreased histamine contents but peptic activity and free acidity of the gastric juice was not affected (Ali and Gerald, 2003).

\subsection{Antimicrobial activity}

On different bacterial isolates, various effects of $\mathrm{N}$. sativa have reported. Both oil and its extracts have reported to having a broad spectrum of activity against various microbes. Such as, pronounced antibacterial activity in vitro have been observed against Shigellaniger, Vibrio cholera, Staphylococcus albus, Escherichia coli, and Salmonella typhi, even in 1:100 dilutions. However, the oil has potent activity against Gram-positive microbes as compared to the Gram-negative microorganism. It has shown that black seeds have strong antifungal activity, especially in Aspergillus species (Salih et al., 2015).

\subsection{Anti-bacterial activity}

It has observed that variety of organisms isolated from human patients suffered from septic arthritis, both water and crude alkaloid extracts of black seeds were effective in different types of microorganisms. Even resistant organisms to antibiotics found to be sensitive to extract of black seeds. Gram-negative microorganism was found to more sensitive than Gram positive. Black seeds exerted their antibacterial action at lower doses than the higher concentration. It has shown that after intraperitoneal administration N. sativa was exhibited strong activity against infection caused by cytomegalovirus in mice. Improvements in innate immunity were related mechanism for antibacterial action of black seeds (Ali and Gerald, 2003). 


\subsection{Anti-parasitic activity}

In comparison to peprazine, $\mathrm{N}$. sativa has shown to have a property of anti-nematodal and anti-cestodal action. The number of worms of a Schistosomamansoni in liver reduced by N. sativa at the dose of 2.5 to $5.0 \mathrm{ml} / \mathrm{kg}$ for 2 weeks gave orally and lessened a total load of ova in both intestine and liver. When praziqauntel given concomitantly with N. sativa, found to reduce the amount of dead ova and diameter of granuloma in the intestinal wall because the activity of praziquantel has potentiated by $\mathrm{N}$. sativa (Salih et al., 2015).

\subsection{Toxicological properties of Nigella sativa}

N. sativa extracts and seeds shown to have a low level of toxicity. Studies have shown that the several enzymes and metabolites are indicative of renal and hepatic function. These enzymes and metabolites not affected by $\mathrm{N}$. sativa at the dose of $50 \mathrm{mg} / \mathrm{kg}$ given intraperitoneally to rats, for 5 days. Under observation period of 48 hours in mice and rats, there was no mortality with $10 \mathrm{ml} / \mathrm{kg}$ after oral administration of $\mathrm{N}$. sativa. The median lethal dose (LD50) of thymoquinone (active component of N. sativa) was observed to be within the range of $1.52-3.77(2.4 \mathrm{~g} / \mathrm{kg})$ was not found to cause any mortality in rats or any change in renal enzyme's function (Ali and Gerald, 2003).

However, after acute administration at the dose of $2 \mathrm{~g} / \mathrm{kg}$ or more than that dose was result in hypo-activity and respiration problems. In fact, these doses depleted the concentration of glutathione stimulating hormone (GSH) in liver, heart and kidney. This proved by the high amount of metabolites and enzyme in plasma. Except for a considerable decrease in fasting plasma glucose level, there were no signs of toxicity in mice when thymoquinone given in drinking water up to $0.03 \%$ for 90 days. Persons who suffered from eczema had allergic contact dermatitis by topically using N. sativa (Ali and Gerald, 2003).

N. sativa oils showed non-specific immune-stimulant effect. Researchers have noted that they have the ability to stimulate responses of immune system, through improving the number of lymphocytes and activities of macrophages both their percent and macrophage index. Studies have shown that it can increase the number of $\mathrm{T}$ cells, activity of natural killer cells, interleukin-IB (IL-IB) and interleukin-3 (IL-3). Researchers were motivated to find out the effect of $\mathrm{N}$. sativa on influenza DNA vaccine (Boskabady et al., 2011).

\subsection{Mechanism of action of Nigella sativa}

Researchers have shown that the thymoquinone and the methanol extract of N. sativa exert significant antimicrobial activity for Gram-positive and Gram-negative bacteria. Phenol compounds of the essential oil sensitize membrane phospholipids, which leads to an increase in the permeability and causes a leakage of intracellular components including bacterial enzyme systems. Based on animal studies, it reported that phagocytic activity and phagocytic index in macrophages of abdomen enhanced by therapy with $\mathrm{N}$. sativa oil. In the case of diabetes, lymphocyte count increased by N. sativa oil. In animals immunosuppressant induced by calcium and lead is retreated by use of N. sativa (Paarakh, 2010).

It was observed in laboratory experiments that expressions of three cytokines from melanin extract of $\mathrm{N}$. sativa have been inducedsuch as Tumor Necrosis Factor-alpha (TNF- alpha), Interleukin-6 (IL-6) and Vascular Endothelial Growth Factor (VEGF). Immunogenicity and tumor suppression promoted by these three cytokines. By altering the level of mediators of inflammation, black seeds have found to improve the immune system (Mady et al., 2013).

Thymoquinone, the major component of N. sativa and fixed oils have shown in liposomes to restrict the non-enzymatic lipid peroxidation. It has also shown that thorough Thin Layer Chromatography (TLC), isolated compound was carvacol, t-anethole, thymoquinone and terpineol. These compounds have shown to have considerable scavenging properties of radicals. These compounds in-vitro experiments have possessed variable antioxidant but, no pro-oxidant properties (Ali and Gerald, 2003).

\subsection{Development of antibiotic resistance in poultry}

It was found that the main risk for incidence of antibiotics resistant strains is wide spread utilization of antibiotics. Different level of resistance has shown by bacteria against antibiotics. For example, the resistance of Encherichia coli and Staphylococcus sp. observed 97\% for tetracycline and $51 \%$ to ampicillin (Apata, 2009)

A technique for gene detection has been ranged from simple Polymerase Chain Reaction (PCR) to techniques based on hybridization such as micro and macro array techniques. The main use of these techniques is to recognize Antibiotic Resistance Genes (ARGs) in samples of bacteria. In bacterial DNA, gene is present as a spot. For identification of ARGs and bacterial genotype, these arrays have used most commonly. For example, glass based developed by a scientist to find out the tetracycline resistance genes. The transfer and spread of Antibiotic Resistance Genes (ARGs) and pathogenic potential of strains of Escherichia coli recognized through this array. Microarrays have also adopted to detect changes in cancerous cells in clinical cytogenetic laboratories. It was also studied that abnormalities in chromosomes have detected by this microarray (McNeece et al.,2014).

\subsection{Infectious Bursal Disease (IBD)}

Worldwide, Infectious Bursal Disease (IBD) has a wide distribution, but there was no reported case of IBD in Ethiopia. This was due to indigenous chicken's resistance to Infectious Bursal Disease Virus and other viruses as well. It was observed that emergence of diseases has a large concern because of rising chicken farming at commercial scale. This reported case observed in 20-45 day old layer and broiler chicks. In these chicks, the level of water and feed consumption dropped rapidly, diarrhea was also a common problem. The prominent clinical symptoms observed; rapid drop of water and feed intake, white creamy diarrhea, mass death, weight loss and soiling of vent and recovered broilers remained underdeveloped. After observable symptoms, high mortality started on the 3rd day and remained continues up to the 15 th day of trial. For both, layers and broiler chicks, the overall mortality rate was $49.89 \%$ at the end of 8 th week. While mortality rates in the layer were $25.1 \%$ and $56.1 \%$ in broiler chicks. In the 3 rd week of age, the disease's onset and appearance of symptoms, both were compatible with the clinical type of the disease (Mohammed et al., 2013).

Widespread malnutrition in women and children is resulted in protein deficiency and poverty in village communities. There were many factors have been associated with low production. Minimal management interventions, feed supplementation, disease control and housing was the part of the system that characterized by low input and low output (Tadesse et al., 2005).

\subsection{New Castle Disease (ND)}

A viral disease called New Castle Disease, chicken mainly affected by it. Urdu name is Rani Khate. It caused by avian paramyxovirus serotype 1 (APMV-1) (NDV) ofthegenus Rubulavirusthatbelongs tothesubfamilyParamyxovirinae, family Paramyxoviridae, order Mononegavirales.The main constraint in low production was different types of diseases, especially New Castle Disease (ND) due to which investments in this system were disturbed. Therefore, by successful control of ND, any improvement for free-range village chickens can make and it can control by vaccination. A disease first reported in Java in 1926 and has endemic in Tanzania. Nevertheless, it was observed that ND is a widespread disease and have different names depending on locality for example "Kideri, Mdonde, Mdondo" in Swahili, while "Kifwa" or "Ikula" in Nyamwezi and Sukuma, respectively. In literature, theirnames were meantplague, fatality, and farmers in Tanzania considered it as the killer disease for village chickens (Mohammed et al., 2013).

Hyperplasia of interfollicular tissue and reticuloendothelial cells, necrosis of lymphoid cells and congestion of vascular layer has observed after histological studies of the affected bursa of poultry. The autopsy studies of pathognomonic lesion of bursa and hemorrhages in the muscles also revealed the same results as studied by different scientists. Precipitations of specific and strong antibodies against Infectious Bursal Disease Virus (IBDV) have shown by serological results of Agar Gel Immuno-diffusion test (AGID). After vaccination against New Castle Disease, different tests performed to find out the antibody titer and low antibody titers log 20-log 23 than that what is expected $(\log 25-\log 29)$, was found in vaccinated chicken. National Veterinary Institute laboratory, have shown that birds after vaccination usually have antibody titer ranging from log 25-log 29. This indicated the depletion of macrophages and lymphocytes by IBDV infection resulting in immunosuppression and ultimately vaccine failure (Tadesse et al., 2005).

\subsection{Gut receptors}

Opiate receptors and their endogenous ligand leucine-enkephalin and methionine-enkephalin, these were first endogenous agonist of opioid receptor. Methionine-enkephalin and leucine-enkephalin were Pentapeptide and found in the gut. Gut motility and secretion regulated by the enteric nervous system, opioid receptor agonist shows their action through this pathway.In human and rodents there are three types of opioid receptors, $\kappa$-(KOR), $\mu$-(MOR), and $\delta$-(DOR) subtypes in the gastrointestinal tract (GIT). Opioid receptor activated by opioid peptide after their release. Opioid receptor controlled motility and secretion of GIT and opioid receptors have different distribution in GIT according to species, GI layers, and GI region (Holzer, 2007).

It has observed that improvements of genetic potential have occurred, due to which 50 growth of broiler chicks has increased each year. As a 
result, each year by an average of 0.75 days, there was a decrease in marketing age of broiler. In developing countries, the broiler with genetic improvements gaining 50 grams per day on an average, but this potential might be double by the same broiler (Mohammed et al., 2013).

In recent times, that standard has established about the performance of broiler. Standard is gaining of $2.5 \mathrm{~kg}$ live weight along with feed conversion ratio of 1.72 at forty-two days of age. It was reported that, with environmentally controlled houses and sheds in different companies, a large number of broiler capacity per house than recommended capacity. Hence, at day 32-33, they started to catch the birds and they gave the first performance to big birds. This practice was resulted in loss of growing period of life of birds as well as they gave extra feeding to females birds because of slow growing (Mohammed et al., 2013).

\section{References:}

Abbasnezhad, A., S. Niazmand, M. Mahmoudabady, M. Soukhtanloo, S.A Rezaee andS.M. Mousavi. 2016. Nigella sativa seed decreases endothelial dysfunction in streptozotocin-induced diabetic rat aorta. Avicenna. J. Phytomed. 1:67-76.

Ali, B. H. and B. Gerald. 2003. Pharmacological and toxicological properties of Nigella sativa.Phytother. Res. 17: 299-305.

Apata, D.F. 2009. Antibiotic Resistance in Poultry. Int. J. Poult. Sci. 4: 404 408.

Bashir, M.U., H.J. Qureshi and T. Saleem.2015. Comparison of antiinflammatory activity of Nigella sativa and diclofenac sodium in albino rats.J.Ayub. Med. Coll. Abbottabad. 3:523-6.

Bogaard, A.E.V, R. Willems, N. London, J. Top and E.E Stobbeningh.2002. Antibiotic resistance of faecal enterococci in poultry, poultry farmers and poutry slaughterers. J. Antimicrob. Chemo.49: 497-505.

Boskabady, M.H., R. Keyhanmanesh, S. Khameneh, Y. Doostdar andM.R Khakzad.2011. Potential immunomodulation effect of the extract of Nigella sativa on ovalbumin-sensitized guinea pigs. J. Zhejiang. Univ. Sci. B. 3:201-9. Burits, M., F. Bucar. 2000. Antioxidant activity of Nigella sativa essential oil. Phytother Res. 5:323-8.

Gould, I.M., 2008.The epidemiology of antibiotic resistance. Int. J. Antimicrob. Agents.10: 1-17.

Holzer, P. 2007. Treatment of opioid-induced gut dysfunction.Expert Opin. Investig.2: 181-194.

Islam, M.H., Z. Ahmad and M.T. Salman. 2015. Neuroprotective effects of Nigella sativa extracts during germination on central nervous system. Pharmacogn. Mag. 1: 182-9.

Jaswal, A., M. Sharma, S. Raghuvanshi, S. Sharma, M.S. Reshi, C. Uthra and S. Shukla.2016. Therapeutic efficacy of Nigella sativa Linn. againstantituberculosis drug-induced hepatic injury in wistar rats.J.
Environ.Pathol.Toxicol. Oncol.1:59-71.

Kaleem, M., D. Kirmani, M. Asif, Q. Ahmed and B. Bano. 2006. Biochemical effects of Nigella sativaL seeds in diabetec rats. Indian J. Exp. Biol. 44: 745748

Mady, W.H., A. Arafa, A.S. Hussein, M.M. Aly and H.M. Madbouly. 2013. Nigella sativa oil as an immunostimulant adjuvant in $\mathrm{H} 5$ based DNA vaccine of H5N1 avian influenza virus. 6: 663-668.

Majdalawieh, A.F., R. Hmaidan and R. Carr. 2010. Nigella sativa modulates splenocyte proliferation, Th1/Th2 cytokine profile, macrophage function and NK anti-tumor activity. J. Ethnopharmacol.2: 268-75.

McNeece, G.,N. Violetta, J.W. Martin, S.G.D. James and J.N. Patrick. 2014. Array based detection of antibiotic resistance genes in Gram negativebacteria isolated from retail poultry meat in the UK and Ireland. Int. J. Food Microbiol.179: 24-32.

Mohammed, M.H., A.H.Z. Abdel, K. Latif, and F.H. Mauida F. H. 2013. Conventional and molecular detection of Newcastle disease and Infectious Bursal Disease in chickens.J. World's Poult. Res.1: 05-12.

Morar, M., B. Kirandeep, W.H. Donald, J. Murray and D.W. Gerard.2009. Structure and mechanism of the lincosamide antibiotic adenylyltransferaseLinB.Structure.17: 1549-1550.

Paarakh, P.M. 2010. Nigella Sativa Linn.- A comprehensive review.Ind. J. Natural products and resources.4: 409-429.

Salih, H.M. Aljabre, M.A. Omar and M.A. Randhawa.2015. Dermatological effects of Nigella sativa.J. Dermatol.\& Dermatologic Surgery. 19: 92-98.

Shakeri, F., Z. Gholamnezhad, B. Mégarbane, R. Rezaee and M.H. Boskabady.2016. Gastrointestinal effects of Nigella sativa and its main constituent, thymoquinone: a review.Avicenna. J. Phytomed. 1:9-20.

Suddek, G.M. 2014. Protective role of thymoquinone against liver damage induced by tamoxifen in female rats.Can. J. Physiol. Pharmacol.8:640-4.

Tadesse, S., A. Hagos and A. Zeleke. 2005. Seroprevalence study of Newcastle disease in local chickens inCentral Ethiopia.Intern. J. Appl. Res. Vet. Med. 3: 25 -

Bourgou, S., K. Riadh, B. Amor, S. Ines, F. Hanen and M. Brahim.2008. Phenolic composition and biological activities of Tunisian Nigella sativa L. shoots and roots. C.R.Biologies.1: 48-55.

Proudfoot, F.G., H.W. Hulan, E.D. Jackson and C.D.C. Salisbury.2007. Effect of lincomycin as a growth promoter for broiler chicks. Br. Poult. Sci. 1: 181187.

Sugiharto, S. 2014. Role of nutraceuticals in gut health and growth performance of poultry.Poult. J. Saudi Soc. Agric. Sci. 10: 1-12.

Oliveiraa, S.D.D., S.F. Fabiana, R.S. Lucianaand B. Adriano. 2005. Antimicrobial resistance in Salmonellaenteritidis strains isolatedfrom broiler carcasses, food, human and poultry-related samples. Int. J. Food Microbiol.97: 297305. 